\title{
Perceived injustice contributes to poor rehabilitation outcomes in individuals who have sustained workplace injuries
}

\author{
Heather Adams \\ Department of Psychology, McGill University, Montreal H3A 1B1, Canada. heather@pdp-pgap.com
}

The experience of unnecessary suffering as a result of another's actions or the experience of irreparable losses are likely to give rise to perceptions of injustice. Until recently, little systematic research had been conducted on the effects of perceptions of injustice on recovery outcomes following injury. It is now becoming clear that justice-related appraisals can have a dramatic impact on the physical and emotional consequences of injury. High levels of perceived injustice have been associated with more severe pain, more severe emotional distress, and more pronounced disability. Research has also pointed to multiple sources of a client's perceptions of injustice including, the person responsible for the accident, the insurance representative, as well as the health care provider. This presentation will summarize what is currently known about the relation between perceived injustice and recovery outcomes. The presentation will also address the processes by which perceptions of injustice might contribute to adverse health and mental health outcomes consequent to injury. Implications for prevention and intervention will be discussed. 